\title{
Tendências do diabetes mellitus no Brasil: o papel da transição nutricional
}

\author{
Trends in diabetes mellitus in Brazil: \\ the role of the nutritional transition
}

Daniela Saes Sartorelli 1

Laércio J oel Franco 1

\footnotetext{
1 Departamento de Medicina Social, Faculdade de Medicina de Ribeirão Preto, Universidade de São Paulo. Av. Bandeirantes 3900, Ribeirão Preto, SP 14049-900, Brasil. Ifranco@fmrp.usp.br
}

Abstract Prevalence of type 2 diabetes mellitus has increased rapidly and is expected to increase even further. In Latin America countries there is an upward trend among the younger age groups, with a major negative impact on the quality of life and burden of the disease for the health care system. Increasing rates of overweight and obesity associated with lifestyle changes and aging of the population are the main contributing factors to the increasing preval ence of type 2 diabetes. Dietary shifts in the Brazilian population (low frequency of fiber-rich foods and heavy consumption of saturated fatty acids and sugars) and sedentary lifestyle are the main risk factors for obesity, type 2 diabetes, and other chronic diseases. Diabetes prevention programs are being promoted in several countries with results showing improvement in quality of Iife. Key words Type 2 Diabetes Mellitus; Prevalence; Nutrition Transition; Food Consumption

Resumo A prevalência do diabetes mellitus tipo 2 tem se el evado vertigi nosamente e espera-se ainda um maior incremento. Na América Latina há uma tendência do aumento da freqüência entre as fai xas etári as mais jovens, cujo impacto negati vo sobre a qualidade de vida e a carga da doença ao sistema de saúde é rel evante. O aumento das taxas de sobrepeso e obesidade associado às al terações do estilo de vida eao envel hecimento populaci onal, são os principais fatores que explicam o crescimento da prevalência do diabetes tipo 2. As modificações no consumo alimentar da população brasileira - baixa freqüência dealimentos ricos em fibras, aumento da proporção de gorduras saturadas e açúcares da di eta - associadas a um estilo de vida sedentário compõem um dos principais fatores etiológi cos da obesi dade, diabetes ti po 2 e outras doenças crônicas. Programas de prevenção primária do diabetes vêm sendo desenvolvidos em diversos países, cujos resultados demonstram um impacto posi tivo sobrea qualidade de vida da população.

Palavras-chave Diabetes Mellitus Tipo 2; Prevalência; Transição Nutricional; Consumo deAlimentos 


\section{Introdução}

A prevalência do diabetes mellitus tipo 2 está aumentando de forma exponencial, adquirindo características epidêmicas em vários países, particularmente os em desenvolvimento. Esta revisão analisa a tendência crescente da importância que o diabetes vem adquirindo como problema de saúde, ressaltando sua situação no Brasil. Também é destacada a contribuição representada pelas al terações da estrutura da dieta, da prática de atividades físicas e o conseqüente aumento da prevalência da obesidade neste processo.

\section{O diabetes nas Américas}

Nas Américas, o número de indivíduos com diabetes foi estimado em 35 milhões para o ano 2000 e projetado para 64 milhões em 2025. Nos países desenvolvidos, o aumento ocorrerá principalmente nas faixas etárias mais avançadas, decorrente do aumento da esperança de vida e do crescimento populacional; nos países em desenvolvimento, o aumento será observado em todas as faixas etárias, principalmente no grupo de 45-64 anos onde sua prevalência deverá triplicar, duplicando nas faixas etárias de 20-44 e 65 e mais anos (King et al., 1998).

Pelo fato do diabetes estar associado a maiores taxas de hospitalizações, a maiores necessidades de cuidados médicos, a maior incidência de doenças cardiovasculares e cerebrovasculares, cegueira, insuficiência renal e amputações não traumáticas de membros inferiores, pode-se prever a carga que isso representará para os sistemas de saúde dos países latinoamericanos, a grande maioria ainda com grandes dificuldades no controle de doenças infecciosas.

A maioria dos países latino-americanos não desenvolve um sistema de vigilância epidemiológica para as doenças crônicas na população adulta, em particular sobre o diabetes mellitus. As informações sobre a prevalência do diabetes derivam de inquéritos realizados esporadicamente e não em bases regulares. Poucos têm uma abrangência nacional, sendo a maioria envolvendo cidades ou regiões.

A Tabela 1 resume alguns dados sobre prevalência do diabetes tipo 2 em alguns países latino-americanos e do Caribe, em anos recentes.

Relevante para o aumento da prevalência do diabetes tipo 2 é o crescente aumento na taxa de sobrepeso e obesidade. Resultados de diversos inquéritos populacionais mostram que a incidência de diabetes aumenta com a média do peso da população (Harris et al., 1987). Interessante notar que a incidência de obesidade em crianças abaixo de 12 anos de idade duplicou na última década nos Estados Unidos (CDC, 1997). As implicações que esse aumento na taxa de obesidade nas crianças representa ainda é incerto, porém com certeza repercutirá nas taxas de incidência de diabetes al guns anos mais tarde. Já se tem descrito que o diabetes tipo 2 está se manifestando mais precocemente, até mesmo na adolescência (ADA, 2000).

\section{O diabetes no Brasil}

Um estudo multicêntrico de base populacional, conduzido em 1988 em nove capitais de estados brasileiros, demonstrou que a prevalência do diabetes e a tolerância à glicose diminuída em população urbana, entre 30 e 69 anos de idade, é de 7,6 e 7,8\%, respectivamente. Os casos de diabetes previamente diagnosticados corresponderam a $54 \%$ dos casos identificados, ou seja, $46 \%$ dos casos existentes desconheciam o diagnóstico, que provavelmente seria feito por ocasião de manifestação de al guma complicação crônica do diabetes (Malerbi \& Franco, 1992).

No Brasil, as cidades das regiões Sul e Sudeste, consideradas de maior desenvolvimento econômico do país, apresentam maiores prevalências de diabetes mellitus e de tolerância à glicose diminuída. Os principais fatores associados à maior prevalência do diabetes no Brasil foram a obesidade, o envelhecimento popuIacional e história familiar de diabetes (Malerbi \& Franco, 1992).

No Brasil, tem-se observado um crescente número nas hospitalizações por diabetes, em proporções superiores às hospitalizações por todas as causas, o que de certa forma, traduz 0 aumento na sua prevalência. Parte da dimensão do problema pode ser evidenciada pelos dados apresentados na Tabela 2, que mostram o aumento da freqüência de internações por diabetes, como causa principal ou associada, no período de 1988 a 1997, na região de Ribeirão Preto, São Paulo (Franco \& Rocha, 2002).

A mortalidade proporcional por diabetes mellitus também tem mostrado um importante crescimento, quando comparada a outras afecções (Lessa, 1992). Há estudos que demonstram que o diabetes como causa de morte tem sido subnotificado, pois os diabéticos geralmente morrem devido às complicações crônicas da doença, sendo estas que figuram como causa do óbito. No Estado de São Paulo, no ano de 1992, o diabetes figurou como causa básica 
Tabela 1

Prevalência de diabetes (\%), com intervalo de confiança de 95\%, ajustada por idade*, em indivíduos na faixa etária de 34-64 anos em alguns países da América Latina, em anos recentes.

\begin{tabular}{|c|c|c|c|c|}
\hline \multirow[t]{2}{*}{ País } & \multirow[t]{2}{*}{ População de referência } & \multicolumn{3}{|c|}{ Prevalência (IC 95\%) } \\
\hline & & Homens & Mulheres & Total \\
\hline México & Cidade do México,1994 & $11,9(8,9-17,2)$ & $17,9(13,5-23,5)$ & $14,9(11,9-18,6)$ \\
\hline Jamaica & Spanish Town, 1999 & $9,3(9,1-17,4)$ & $18,4(14,9-22-8)$ & $15.6(13,1-18,6)$ \\
\hline Trinidad e Tobago & St. J ames, 1988 & $9,3(7,7-10,9)$ & $12,5(10,4-14,5)$ & $10,9(9,6-12,1)$ \\
\hline Bolívia & 4 cidades, 2001 & $9,5(7,2-11,8)$ & $10,6(8,6-12,7)$ & $10,1(8,5-11,6)$ \\
\hline Brasil & 9 cidades, 1992 & $6,0(4,8-7,6)$ & $9,7(7,7-12,1)$ & $7,9(6,7-9,2)$ \\
\hline Chile & Santiago, 1983 & $10,1(5,9-16,1)$ & $6,7(4,3-10,0)$ & $8,4(6,0-11,4)$ \\
\hline Colômbia & Bogotá, 1992 & $7,6(4,1-12,8)$ & $8,6(4,7-14,4)$ & $8,1(5,3-11,8)$ \\
\hline Paraguai & Assunção, 1998 & $7,6(4,9-11,3)$ & $8,6(6,2-10,9)$ & $8,9(6,3-10,0)$ \\
\hline
\end{tabular}

* Taxas ajustadas usando a população mundial de Segi, pelo método direto.

Fonte: Barceló \& Rajpathak (2001).

em $4,0 \%$ dos óbitos e como causa mencionada em $10,1 \%$ dos atestados de óbito (Franco et al., 1998).

\section{A transição nutricional}

\section{Alterações na estrutura da dieta}

As alterações na estrutura da dieta, associadas a mudanças econômicas, sociais e demográficas e suas repercussões na saúde populacional, vêm sendo observadas em diversos países em desenvolvimento (Popkin, 2001).

Conforme Monteiro et al. (2000a), no período entre 1988 e 1996, observou-se um aumento do consumo de ácidos graxos saturados, açúcares e refrigerantes, em detrimento da redução do consumo de carboidratos complexos, frutas, verduras e legumes, nas regiões metropolitanas do Brasil.

Dados sobre o consumo de ácidos graxos "trans", encontrados principalmente nas margarinas, alimentos tipo fast-foods e outros produtos industrializados, ainda são escassos. Entretanto, conforme Mondini \& Monteiro (1995), entre 1962 e 1988 o consumo de margarina no Brasil subiu de 0,4 para $2,5 \%$ do total de calorias. Observou-se também, um incremento da densidade energética, favorecido pelo maior consumo de carnes, leite e derivados ricos em gorduras.

A crescente substituição dos alimentos in natura ricos em fibras, vitaminas e minerais, por produtos industrializados (Barreto \& CyrilIo, 2001), associada a um estilo de vida sedentário, favorecido por mudanças na estrutura de
Tabela 2

Evolução do crescimento populacional e das hospitalizações por todas as causas e por diabetes na região de Ribeirão Preto, São Paulo, Brasil, no período de 1988 e 1997.

\begin{tabular}{lrrc}
\hline & \multicolumn{2}{c}{ Número total } & $\begin{array}{c}\text { Proporção de } \\
\text { aumento (\%) }\end{array}$ \\
& 1988 & 1997 & \\
\hline Hospitalizações & & & \\
$\quad$ Todas as causas & 128.181 & 146.460 & 14,3 \\
$\quad$ Diabetes como causa principal & 1.050 & 1.403 & 33,6 \\
Com menção de diabetes & 2.438 & 3.751 & 53,9 \\
População & 822.027 & 991.975 & 20,6 \\
\hline
\end{tabular}

Fonte: Franco \& Rocha (2002).

trabalho e avanços tecnológicos (Popkin, 1999), compõem um dos os principais fatores etiológicos da obesidade.

\section{A redução da atividade física}

Algumas evidências sugerem que o sedentarismo, favorecido pela vida moderna, é um fator de risco tão importante quanto a dieta inadequada na etiologia da obesidade (Prentice $\&$ Jebb, 1995), e possui uma relação direta e positiva com o aumento da incidência do diabetes tipo 2 em adultos, independentemente do índice de massa corporal (Manson et al., 1991), ou de história familiar de diabetes (Zimmet et al., 1997).

Alguns estudos demonstram que o controle de peso e aumento da atividade física dimi- 
nuem a resistência à insulina, diminuindo as chances de se desenvolver o diabetes mellitus (Pan et al., 1997). A prática de atividades físicas regulares promove um aumento do turnover da insulina por maior captação hepática e melhor sensibilidade dos receptores periféricos (Oshida et al., 1989). Além disso, a prática de atividades físicas, associada à dieta, melhora o perfil lipídico de indivíduos em risco de desenvolvimento de doenças cardiovasculares (Stefanick et al., 1998).

No Brasil, a redução do nível de atividade física tem sido atribuída à modernização dos processos produtivos, inclusive na agricultura, observado nas últimas décadas (INAN, 1991). Estudos realizados no Estado do Rio de Janeiro, demonstraram que mulheres e indivíduos de baixa escolaridade tendem a praticar atividades físicas com menor freqüência (Gomes et al., 2001), e entre adolescentes do sexo masculino, o número de horas gastas com televisão/ “vídeo-game", tidos como atividades sedentárias, estava associado positivamente com o índice de massa corporal (Fonseca et al., 1998).

\section{O incremento da obesidade}

A prevalência de desnutrição diminuiu consideravelmente nas últimas décadas em adultos e crianças. Por outro lado, uma evolução positiva na prevalência da obesidade vem sendo observada em homens e mulheres (Monteiro et al., 1995). Em áreas urbanas, a prevalência da obesidade é predominante nas classes sociais menos favorecidas (M onteiro et al., 2000b), e as taxas entre mulheres de maiores extratos econômicos mostraram uma redução na última década (Monteiro et al., 2001), apontando um maior impacto do excesso de peso entre as classes sociais mais baixas.

Em muitos países, a prevalência da obesidade ou sobrepeso tem se elevado vertiginosamente. A Organização Mundial da Saúde, em seu relatório de 1997, alerta sobre a epidemia global de obesidade (WHO, 1997), e da necessidade de prevenção primária do excesso de peso.

O aumento abrupto da prevalência da obesidade observado em países do continente americano nas últimas décadas, tem sido atribuído a al terações do consumo alimentar - maior densidade energética, alto consumo de carboidratos refinados, gorduras saturadas, ácidos graxos "trans", colesterol, bebidas alcoólicas e alimentos tipo fast-foods; redução do gasto energético - avanços tecnológicos no trabalho, uso de veículo automotor, escadas rolantes, elevadores, aumento do tempo gasto em atividades sedentárias (televisão, "vídeo-games", computadores), assim como fatores socioculturais - valorização do excesso de peso como sinônimo de saúde e prosperidade (Peña \& Bacallao, 2001).

A obesidade tem sido apontada como um dos principais fatores de risco para o diabetes tipo 2. Estima-se que entre 80 e $90 \%$ dos indivíduos acometidos por esta doença são obesos e o risco está diretamente associado ao aumento do índice de massa corporal.

\section{Discussão}

Em muitos países, a prevalência do diabetes mellitus tem se elevado vertiginosamente e espera-se ainda um maior incremento. Nos países em desenvolvimento há uma tendência de aumento na freqüência em todas as faixas etárias, especialmente nas mais jovens, cujo impacto negativo sobre a qualidade de vida e a carga da doença aos sistemas de saúde é imensurável.

O diabetes se associa a grandes cargas econômicas e sociais, tanto para o indivíduo como para a sociedade. Seus custos estão relacionados principalmente com uma alta freqüência de complicações agudas e crônicas, que são causas de hospitalização, incapacitações, perda de produtividade de vida e morte prematura (Harris, 1998).

O número crescente de indivíduos acometidos por esta doença tem sido associado às rápidas transformações demográficas e sócioeconômicas observadas em vários países em desenvolvimento, como por exemplo China e Cingapura (Cavalli-Sforza et al., 1996; Popkin et al., 1995). A grande variação da prevalência do diabetes em diferentes nações tem sido atribuída a uma combinação de diferenças genéticas e fatores ambientais, como dieta, obesidade, sedentarismo e desenvolvimento intra-uterino (Zimmet et al., 1997).

Os hábitos al imentares e a prática de atividades físicas exercem uma poderosa influência sobre o balanço energético, sendo considerados os principais fatores, passíveis de modificação, determinantes da obesi dade. Dietas com alta densi dade energética associadas a um estilo de vida sedentário, são apontados como os principais fatores etiológicos do aumento da prevalência da obesidade no mundo (WHO, 1997).

Há evidências bem fundamentadas da relação entre a qualidade da alimentação e os riscos de desenvolver o diabetes mellitus. Tem sido demonstrada uma correlação positiva entre a prevalência do diabetes e o alto consumo de gorduras saturadas e ao baixo teor de fibras da dieta (Feskens \& Kromhout, 1990). 
As mudanças observadas no consumo alimentar no Brasil, com especial destaque para o aumento da densidade energética, maior consumo de carnes, leite e derivados ricos em gorduras e redução do consumo de cereais (Mondini \& Monteiro, 1995), frutas, verduras e legumes (Monteiro et al., 2000a), constituem um importante fator de risco para o desenvolvimento do diabetes, independentemente do índice de massa corporal (Gittelsohn et al., 1998).

$O$ aumento do consumo de ácidos graxos "trans" no Brasil, favorecido pela maior ingestão de margarinas, parece ser um fator de risco ainda mais importante do que o consumo de gorduras saturadas para o desenvolvimento de doenças cardiovasculares (Aschiero et al., 1999).

A qualidade dos lipídeos possui um papel importante no risco de desenvolvimento do diabetes. Estudos prospectivos demonstram correlação positiva entre consumo de gorduras saturadas e os níveis de glicemia (Feskens \& Kromhout, 1990), maior risco de progressão de tolerância à gli cose diminuída para o diabetes (Tsunehara et al., 1991) e uma correlação negativa com o consumo de ácidos graxos $\omega$-3 (Feskens et al., 1991).

No Brasil, um estudo realizado na comunidade nipo-brasileira de Bauru, verificou um maior consumo de calorias provenientes dos lipídeos em relação aos hábitos da população do Japão (Freire, 2002). Nessa população nipobrasileira, a prevalência do diabetes tipo 2 , tolerância à glicose diminuída e glicemia de jejum alterada vem aumentando vertiginosamente nos últimos anos (Tabela 3), demonstrando o impacto de condições ambientais interagindo com uma provável susceptibilidade genética sobre os riscos de desenvolver alterações no metabolismo da glicose (Gimeno et al., 2000b).

A redução do consumo de frutas, verduras e legumes, observada por Monteiro et al. (2001a), pode favorecer o aumento da prevalência das doenças crônicas no Brasil, já que o consumo desses alimentos tem sido apontado como um fator protetor para o desenvolvimento de doenças cardiovasculares arteroscleróticas (J oshpura et al., 1999; Wolk et al., 1999) e outras doenças crônicas (Willett, 1994).

Estudos recentes sobre padrões alimentares têm demonstrado que o consumo habitual da dieta "ocidental", caracterizada por uma alta ingestão de carnes vermelhas, produtos lácteos integrais, bebidas adocicadas, açúcares e sobremesas, está diretamente relacionado ao risco de desenvolver obesidade, doenças cardiovasculares (Fung et al., 2001) e diabetes
Tabela 3

Prevalência do diabetes tipo 2, tolerância à glicose diminuída e glicemia de jejum alterada, na comunidade nipo-brasileira de Bauru nos anos de 1993 (40-79 anos) e 1999 (30-87 anos). Bauru, São Paulo, Brasil.

\begin{tabular}{lcc}
\hline Classificação da & \multicolumn{2}{c}{ Prevalência (\%) } \\
homeostase glicêmica* & $1993(\mathrm{n}=530)$ & $1999(\mathrm{n}=1.700)$ \\
\hline Diabetes tipo 2 & 18,3 & 34,9 \\
Tolerância à glicose diminuída & 16,8 & 26,9 \\
Glicemia de jejum alterada & 3,2 & 18,2 \\
\hline
\end{tabular}

Fonte: Gimeno et al. (2000b).

* Classificação da homeostase glicêmica: diabetes tipo 2 (glicose jejum $\geq 126 \mathrm{mg} / \mathrm{d}$ l e/ou glicemia pós-carga $\geq 200 \mathrm{mg} / \mathrm{dl}$ ); tolerância à glicose diminuída (glicemia de jejum $<126$ e glicemia pós-carga $\geq 140$ e $<200 \mathrm{mg} /$ dl) e glicemia de jejum alterada (glicemia de jejum $\geq 110$ e $<126$, e glicemia pós-carga $<140 \mathrm{mg} / \mathrm{dl}$ ).

(Gittelsohn et al., 1998). Em contrapartida, um padrão alimentar mais saudável, rico em frutas, verduras, legumes e peixes, associado ao consumo infreqüente de frituras e embutidos, demonstrou ser um fator protetor para o desenvolvimento de tolerância à glicose diminuída e da síndrome metabólica (Williams et al., 2000).

As mudanças do perfil de atividade física da população e disseminação de atividades sedentárias, devido à modernização dos processos produtivos (INAN, 1991), e maior acesso à tecnologia também possuem um impacto negativo para a saúde. O sedentarismo tem sido apontado como um importante fator de risco para a obesidade (Prentice \& Jebb, 1995), diabetes (Baan et al., 1999; Manson et al., 1991) e doenças cardiovasculares (Lakka et al., 1994). Estudos de base populacional neste sentido são escassos no Brasil, entretanto uma pesquisa realizada na cidade do Rio de Janeiro, sugere que o sedentarismo é mais freqüente entre mulheres e indivíduos de baixa escolaridade (Gomes et al., 2001).

As implicações do aumento da prevalência da obesidade observada em homens e mulheres (Monteiro et al., 1995), especialmente nas classes sociais menos favorecidas (Monteiro et al., 2000b), são de grande amplitude para a saúde pública no Brasil. As evidências dos riscos de desenvolvimento de diabetes, tolerância à glicose diminuída (Gimeno et al., 2000a) e outras doenças crônicas não transmissíveis (Field et al., 2001; Willett et al., 1999), associadas ao excesso de peso, especialmente na região abdominal (Bloch, 1998), há muito vêm sendo documentadas. Um ganho de peso na vida adulta de $5 \%$ em relação ao peso referido aos 20 anos de idade está relacionado à maior ocorrência 
de hipertensão, dislipidemia e, principalmente, hiperinsulinemia (Everson et al., 1998).

As evidências apontadas sobre as alterações no estilo de vida, com ênfase na alimentação e prática de atividades físicas, associadas ao aumento da esperança de vida dos brasileiros são apontados atualmente como os principais fatores responsáveis pelo aumento da prevalência do diabetes tipo 2 observado no país. Portanto, a importância da prevenção primária de obesi dade e diabetes no Brasil tem sido enfatizada por diversos epidemiologistas.

Em várias partes do mundo, programas de prevenção do diabetes vêm sendo implementados com o intuito de se avaliar as formas mais eficientes de prevenção ou retardo do aparecimento do diabetes mellitus tipo 2 em indivíduos portadores de fatores de risco (Bourn et al., 1994; Diabetes Prevention Program Group, 1999; Pan et al., 1997; Wing et al., 1998).

Os resultados do Diabetes Prevention Program demonstraram uma redução de $58 \%$ da incidência dos casos de diabetes por meio do estímulo à dieta saudável e prática de atividades físicas, sendo essa medida significativamente mais efetiva do que o uso da metformina na prevenção primária do diabetes tipo 2 (Diabetes Prevention Program Group, 2002). Tuomilehto et al. (2001) observaram que a perda de peso em torno de 3 a $4 \mathrm{~kg}$ em 4 anos reduziu em $58 \%$ a incidência do diabetes em população de alto risco. O controle de alguns fatores de

Referências

ADA (American Diabetes Association), 2000. Type 2 diabetes in children and adolescents. Diabetes Care, 23:381-389.

ASCHIERO, A.; KATAN, M. B.; ZOCK, P. L.; STAMPFER, M. J . \& WILLETT, W. C., 1999. Trans fatty acids and coronary heart disease. New England Journal of Medicine, 340:1994-1998.

BAAN, C. A.; STOLK, R. P.; GROBEE, D. E.; WITTEMAN, J. C. M. \& FESKENS, J. M., 1999. Physical activity in elderly subjects with impaired glucose tolerance and newly diagnosed diabetes mellitus. American Journal of Epidemiology, 149:219-227.

BARCELÓ, A. \& RAJPATHAK, S., 2001. Incidence and prevalence of diabetes mellitus in the Americas. Revista Panamericana de Salud Pública, 10:300308.

BARRETO, S. A. J. \& CYRILLO, D. C., 2001. Análise da composição dos gastos com alimentação no Município de São Paulo (Brasil) na década de 1990. Revista de Saúde Pública, 35:52-59. risco modificáveis, como o peso, consumo alimentar habitual, uso do tabaco e prática de atividades físicas mostrou possuir um potencial de redução de $88 \%$ no risco de desenvolver o diabetes em indivíduos com história familiar (Hu et al., 2001).

Entretanto, diferenças sociais, econômicas e culturais podem influenciar os desfechos de programas de prevenção, sendo necessário testar sua eficácia em diferentes populações. No Brasil, estudos sobre impacto de programas de prevenção primária de diabetes tipo 2 em população saudável ou de alto risco são escassos. Dados preliminares de um estudo de intervenção para a prevenção do diabetes em população adulta sugerem que esses programas são viáveis em unidades bási cas de saúde (Sartorelli et al., 2001), e promovem a melhoria da saúde na população.

Há evidências de que mudanças de estilo de vida possam ocorrer com maior sucesso quanto mais precoce forem as intervenções (Wing et al., 1998), e não há controvérsias que a adoção de uma alimentação saudável, rica em frutas, verduras, legumes, grãos integrais e pobre em gorduras saturadas (Willett, 1994), associada à prática freqüente de atividades físicas, no mínimo trinta minutos ao dia, possam atuar beneficamente na qualidade de vida da população e na carga de doenças ao sistema de saúde pública.
BLOCH, K. V., 1998. Fatores de risco cardiovasculares e para o diabetes mellitus. In: O Adulto Brasileiro eas Doenças da Modernidade: Epidemiologia das Doenças Crônicas Não-Transmissíveis (I. Lessa, org.), pp. 43-72, São Paulo: Editora Hucitec/Rio de Janeiro: ABRASCO.

BOURN, D. M.; MANN, J. I.; MCSKIMMING, B. J.; WALDRON, M. A. \& WISHRT, J. D., 1994. Impaired glucose tolerance and NIDDM: Does a lifestyle intervention program have an effect? Diabetes Care, 17:1311-1319.

CAVALLI-SFORZA, L. T.; ROSMAN, A.; DE BOER, A. S. \& DARNTON-HILL, I., 1996. Nutritional aspects of changes in disease patterns in the Western Pacific Region. Bulletin of the World Health Organization, 74:307-318.

CDC (Centers for Disease Control and Prevention), 1997. Update: Prevalence of overweight among children, adolescents, and adults - United States, 1988-1994. JAMA, 227:1111. 
DIABETES PREVENTION PROGRAM GROUP, 1999. The Diabetes Prevention Program. Design and methods for a clinical trial in the prevention of type 2 diabetes. Diabetes Care, 22:623-634.

DIABETES PREVENTION PROGRAM GROUP, 2002. Reduction in the incidence of type 2 diabetes with lifestyle intervention or metformin. New England Journal of Medicine, 346:393-403.

EVERSON, S. A.; GOLDBERG, D. E.; HELMRICH, S. P.; LAKKA, T. A.; LYNCH, J. W.; KAPLAN, G. A. \& SALONEN, J. T., 1998. Weight gain and the risk of developing Insulin Resistance Syndrome. Diabetes Care, 21:1637-1643.

FESKENS, E. J. M. \& KROMHOUT, D., 1990. Habitual dietary intake and glucose tolerance in euglycaemic men: The Zutphen Study. International Journal of Epidemiology, 19:953-959.

FESKENS, E. J. M.; BOWLES, C. H. \& KROMHOUT, D., 1991. Inverse association between fish intake and risk of glucose intolerance in normoglycemic elderly men and women. Diabetes Care, 14:935-941.

FIELD, A. E.; COAKLEY, E. H.; MUST, A.; SPADANO, J. L.; LAIRD, N.; DIETZ, W. H.; RIMM , E. \& COLDITZ, G. A., 2001. Impact of overweight on the risk of developing common chronic disease during a 10year period. Archives of Internal Medicine, 161: 1581-1586.

FONSECA, V. M.; SICHIERI, R. \& VEIGA, G. V., 1998. Fatores associados à obesidade em adolescentes. Revista de Saúde Pública, 32:541-549.

FRANCO, L. J.; MAMERI, C.; PAGLIARO, H.; IOCHIDA, L. C. \& GOLDENBERG, P., 1998. Diabetes como causa básica ou associada de morte no Estado de São Paulo, Brasil, 1992. Revista de Saúde Pública, 32:237-245.

FRANCO, L. J. \& ROCHA, J. S. Y., 2002. O aumento das hospitalizações por diabetes na região de Ribeirão Preto, SP, no período de 1988-97. Diabetes Clínica, 6:108.

FREIRE, R. D., 2002. Estado Nutricional de Nipo-Brasilei ros de Alto Risco para Síndrome Metabólica: Análise por Sexo e Geração. Dissertação de Mestrado, São Paulo: Escola Paulista de Medicina, Universidade Federal de São Paulo.

FUNG, T. T.; RIMM, E. B.; SPIEGELMAN, D.; RIFAI, N.; TOFLER, G. H.; WILLETT, W. C. \& HU, F. B., 2001. Association between dietary patterns and plasma biomarkers of obesity and cardiovascular disease risk. American Journal of Clinical Nutrition, 73: 61-67.

GIMENO, S. G. A.; FERREIRA, S. R. G.; CARDOSO, M. A.; FRANCO, L. J.; IUNES, M. \& JAPANESE-BRAZILIAN STUDY GROUP, 2000a. Weight gain in adulthood and risk of developing glucose tolerance disturbance: A study of Japanese-Brazilian Population. Journal of Epidemiology, 10:103110.

GIMENO, S. G. A.; FERREIRA, S. R. G.; FRANCO, L. J. \&IUNES, M., 2000b. 7-Yr incidence of glucose intolerance in a population-based study of Japanese-Brazilians: Preliminary data. Diabetes Research and Clinical Practice, 50(Sup. 1):121.

GITTELSOHN, J.; WOLEVER, T. M. S.; HARRIS, S. B.; HARRIS-GIRALDO, R.; HANLEY, A. J. G. \& ZINMAN, B., 1998. Specific patterns of food consumption and preparation are associated with di- abetes and obesity in a native Canadian community. Journal of Nutrition, 128:541-547.

GOMES, V. B.; SIQUEIRA, K. S. \& SICHIERI, R., 2001. Atividade física em uma amostra probabilística da população do Município do Rio de Janeiro. Cadernos de Saúde Pública, 17:969-976.

HARRIS, M. I., 1998. Diabetes in America: Epidemiology and scope of the problem. Diabetes Care, 21 (Sup. 3):11-14.

HARRIS, M. I.; HADEN, W. C.; KNOWLER, W. C. \& BENNETT, P. H., 1987. Prevalence of diabetes and impaired glucose tolerance and plasma glucose levels in US population aged 20-74 Yr. Diabetes, 36:523-534.

HU, F. B.; MANSON, J. E.; STAMPFER, M. J.; COLDITZ, G.; LIU, S.; SOLOMON, C. G. \& WILLETT, W. C., 2001. Diet, lifestyle, and the risk of type 2 diabetes mellitus in women. New England Journal of Medicine, 345:790-797.

INAN (Instituto Nacional de Alimentação e Nutrição), 1991. Condições nutricionais da população brasileira: Adultos e idosos. In: Pesquisa Nacional sobreSaúdee Nutrição (M inistério da Saúde - MS, org.), p. 32, Brasília: INAN/MS.

JOSHIPURA, K. J.; ASCHERIO, A.; MANSON, J. E.; STAMPFER, M. J.; RIMM, E. B.; SPEIZER, F. E.; HENNEKENS, C. H.; SPIELM AN, D. \& WILLETT, W. C., 1999. Fruit and vegetable intake in relation to risk of ischemic stroke. JAMA, 282:1233-1239.

KING, H.; AUBERT, R. E. \& HERMAN, W. H., 1998 Global burden of diabetes, 1995-2025. Diabetes Care, 21:1414-1431.

LAKKA, T. A.; VENALAINEN, J. M.; RAURAMAA, R.; SALONEN, R.; TUOMILEHTO, J. \& SALONEN, J., 1994. Relation of leisure-time physical activity and cardio-respiratory fitness to the risk of acute myocardial infarction in men. New England Journal of Medicine, 330:1549-1554.

LESSA, I., 1992. Tendência da mortalidade proporcional pelo diabetes mellitus nas capitais brasileiras, 1950-1985. Boletín dela Oficina Sanitaria Panamericana, 113:212-217.

MALERBI, D. A. \& FRANCO, L. J., 1992. Multicenter study of the prevalence of diabetes mellitus and impaired glucose tolerance in the urban Brazilian population aged 30-69 Yr. Diabetes Care, 15:15091516.

MANSON, J. E.; RIMM, E. B.; STAMPFER, M. J.; COLDITZ, G. A.; WILLETT, W. C.; KROLEWSKI, A. S.; ROSNER, B.; HENNEKENS, C. H. \& SPEIZER, F. E., 1991. Physical activity and incidence of noninsulin-dependent diabetes mellitus in women. Lancet, 338:774-778.

MONDINI, L. \& MONTEIRO, C. A., 1995. Mudanças no padrão de alimentação. In: Vel hos e Novos Males da Saúde no Brasil. A Evolução do País e de suas Doenças (C. A. Monteiro, org.), pp. 79-89, São Paulo: Editora Hucitec/Núcleo de Pesquisas Epidemiológicas em Nutrição e Saúde, Universidade de São Paulo.

MONTEIRO, C. A.; BENICIO, M. H.; CONDE, W. L. \& POPKIN, B. M., 2000b. Shifting obesity trends in Brazil. European Journal of Clinical Nutrition, 54:342-346.

MONTEIRO, C. A.; CONDE, W. L. \& POPKIN, B. M., 2001. Independent effects of income and educa- 
tion on the risk of obesity in Brazilian adult population. Journal of Nutrition, 131: 881-886.

MONTEIRO, C. A.; MONDINI, L. \& COSTA, R. L., 2000a. Mudanças na composição e adequação nutricional da dieta familiar nas áreas metropolitanas do Brasil (1988-1996). Revista de Saúde Pública, 34:251-258.

MONTEIRO, C. A.; MONDINI, L.; DE SOUZA, A. L. \& POPKIN, B. M., 1995. The nutrition transition in Brazil. European Journal of Clinical Nutrition, 49:105-113.

OHIDA, Y.; YAM ANOUCHI, K.; HAYAMIZU, S. \& SATO, Y., 1989. Long-term mild jogging increases insulin action despite no influence on body mass or $\mathrm{VO}_{2}$ max. Journal of Applied Physiology, 66: 2206-2210.

PAN, X. R.; LI, G. W.; HU, Y. H.; WANG, J. X.; YANG, W. Y.; AN, Z. X.; HU, Z. X.; LIN, J.; XIAO, J. Z.; CAO, H. B. C.; LIU, P. A.; JIANG, X. G.; WANG, J. P.; ZHENG, H.; ZHANG, H.; BENNETT, P. H. \& HOWARD, B. V., 1997. Effect of diet and exercise in preventing NIDDM in people with impaired glucose tolerance. Diabetes Care, 20:537-544.

PEÑA, M. \& BACALLAO, J., 2001. La obesidad y sus tendencias en la región. Revista Panamericana de Salud Pública, 10:75-78.

POPKIN, B. M., 1999. Urbanization, lifestyle changes and the nutrition transition. World Development, 27:1905-1916.

POPKIN, B. M., 2001. The nutrition transition and obesity in the developing world. Journal of Nutrition, 131:871-873.

POPKIN, B. M.; PAERATAKUL, S.; ZHAI, F. \& GE, K., 1995. A review of dietary and environmental correlates of obesity with emphasis on developing countries. Obesity Research, 3(Sup. 2):145-153.

PRENTICE, A. M. \& JEBB, S. A., 1995. Obesity in Britain: Gluttony or sloth? BMJ, 311:437-439.

SARTORELLI, D. S.; MONTEIRO, R. C.; SCIARRA, E. C.; FRIZZAS, P. E.; FRANCO, L. J. \& CARDOSO, M. A., 2001. Intervenções nutricionais para prevenção do diabetes tipo 2 em adultos com sobrepeso: Dados preliminares. Arquivos Brasileiros de Endocrinologia e Metabologia, 45:560.

STEFANICK, M. L.; MACKEY, S.; SHEEHAN, M.; ELLSWORTH, N.; HASKELL, W. L. \& WOOD, P. D., 1998. Effects of diet and exercise in men and postmenopausal women with low levels of HDL cholesterol and high levels of LDL cholesterol. New England Journal of Medicine, 339:12-20.
TSUNEHARA, C. H.; LEONETTI, D. L. \& FUJIMOTO, W. Y., 1991. Animal fat and cholesterol intake is high in men with IGT progressing to NIDDM. Diabetes, 40:427A.

TUOMILEHTO, J.; LINDSTM, J.; ERIKSSON, J. G.; VALLE, T. T.; HAM ALAINEN, H.; HANNE-PARIKKA, P.; KEINANEN-KIUKAANNIEMI, S.; LAASKO, M.; LOU HERANTA, A.; RASTAS, M.; SALMINEM, V. \& UUSITUPA, M., 2001. Prevention of type 2 diabetes mellitus by changes in lifestyle among subjects with impaired glucose tolerance. New England Journal of Medicine, 344:1343-1350.

WHO (World Health Organization), 1997. Obesity: Preventing and Managing the Global Epidemic. Report of a WHO consultation group on obesity. Geneva: WHO.

WILLETT, W. C., 1994. Diet and health: What should we eat? Science, 264:532-537.

WILLETT, W. C.; DIETZ, W. H. \& COLDITZ, G. A., 1999. Guidelines for health weight. New England Journal of Medicine, 341:427-434.

WILLIAMS, D. E. M.; PREVOST, A. T.; WHICHELOW, M. J.; COX, B. D.; DAY, N. E. \& WAREHAM, N. J., 2000. A cross-sectional study of dietary patterns with glucose intolerance and other features of the metabolic syndrome. British Journal of Nutrition, 83:257-266.

WING, R. R.; VENDITTI, E.; JAKICIC, J. M.; POLLEY, B. A. \& LANG, W., 1998. Lifestyle intervention in overweight individual with a family history of diabetes. Diabetes Care, 21:350-359.

WOLK, A.; MANSON, J. E.; STAMPFER, M. J.; COLDITZ, G. A.; HU, F. B.; SPEIZER, F. E.; HENNEKENS, C. H. \& WILLETT, W. C., 1999. Longterm intake of dietary fiber and decreased risk of coronary heart disease among women. JAMA, 281:1998-2004.

WCRF (World Cancer Research Fund)/ AICR (American Institute for Cancer Research), 1997. Food, Nutrition and the Prevention of Cancer: A Global Perspective. Washington, DC: WCRF/AICR.

ZIMMET, P. Z.; M CCARTY, D. J. \& COURTEN, M. P., 1997. The global epidemiology of non-insulin-dependent diabetes mellitus and the metabolic syndrome. Journal of Diabetes and Its Complications, 11:60-68.

Recebido em 12 de junho de 2002

Versão final reapresentada em 17 de setembro de 2002 Aprovado em 5 de novembro de 2002 